\title{
Effect of non-sentinel metastasis on adjuvant treatment decisions and survival in Z0011 eligible non-screened detected breast cancer population
}

\author{
Sanjit Kumar Agrawal ${ }^{1}$, Vishal Kewlani ${ }^{1}$, Noopur Priya ${ }^{1}$, Abhishek Sharma ${ }^{1}$, Joydeep Ghosh ${ }^{2}$, Sanjoy Chatterjee ${ }^{3}$, Rosina Ahmed ${ }^{1}$ \\ ${ }^{1}$ Department of Breast Oncosurgery, Tata Medical Center, 14, MAR(E-W), DH Block(Newtown), Action Area I, Newtown, Kolkata, West Bengal 700160, India \\ ${ }^{2}$ Department of Medical Oncology, Tata Medical Center, 14, MAR(E-W), DH Block (Newtown), Action Area I, Newtown, Kolkata, West Bengal 700160, \\ Kolkata, India \\ ${ }^{3}$ Department of Clinical Oncology, Tata Medical Center, 14, MAR(E-W), DH Block(Newtown), Action Area I, Newtown, Kolkata, West Bengal 700160, \\ Kolkata, India
}

\begin{abstract}
Background: The Z0011 trial results have shown that axillary lymph node dissection (ALND) can be avoided in cT1-2 patients undergoing breast conservation surgery with 1-2 metastatic sentinel lymph nodes (SLNs). We compared the clinicopathological characteristics of the Z0011 eligible non-screen detected breast cancer patients' cohort with the Z0011 trial study population. Additionally, we have explored the effect of non-sentinel metastasis on adjuvant treatment decisions and survival.
\end{abstract}

Methods: The details of early breast cancer (EBC) patients fulfilling Z0011 eligibility criteria were retrieved from a prospectively maintained database (2013-2017) and electronic medical records. We used Statistical Package for the Social Sciences 25 and Stata V15 for the data analysis.

Results: 128/194 (66\%) sentinel lymph node biopsy positive patients had fulfilled the Z0011 inclusion criteria. Compared to the Z0011 study, our cohort patients were younger, with more aggressive disease (higher T2, Grade 3), had a higher rate of macrometastasis ( $82.8 \%$ versus $58.8 \%$ ) and non-SLN metastasis ( $48 \%$ versus $27 \%$ ). The information gained by ALND had changed decisions for chemotherapy in $3 \%$ and no change of radiotherapy in Z0011 eligible patients. Further nodal positivity in completion ALND was not significantly associated with overall survival $(p=0.86)$ and disease-free survival $(p=0.5)$.

Conclusion: Z0011 eligible Indian EBC patients are significantly different from the Z0011 study population, with younger age of presentation, higher grade, a higher rate of both SLN macro metastasis and non-SLN positivity. The impact of non-sentinel metastasis on adjuvant treatment decisions and survival is minimal.

Keywords: breast cancer, SLNB, Z0011, India

\section{Introduction}

In the last three decades, surgical management of axilla has evolved from axillary lymph node dissection (ALND) to sentinel lymph node biopsy (SLNB) in the majority of breast
Correspondence to: Sanjit Kumar Agrawal Email: sanjitgrwl@gmail.com

ecancer 2021, 15:1324

https://doi.org/10.3332/ecancer.2021.1324

Published: $26 / 11 / 2021$

Received: 04/07/2021

Publication costs for this article were supported by ecancer (UK Charity number 1176307).

Copyright: $($ ) the authors; licensee ecancermedicalscience. This is an Open Access article distributed under the terms of the Creative Commons Attribution License (http:// creativecommons.org/licenses/by/4.0), which permits unrestricted use, distribution, and reproduction in any medium, provided the original work is properly cited. 
cancer patients [1-4]. SLNB alone reduces the morbidity of ALND procedure like shoulder stiffness, pain and paresthesia in the arm and risk of lymphedema [1]. Before 2010, ALND was the standard procedure for patients with metastatic sentinel lymph nodes (SLN). However, in the last decade, three randomised controlled trials (Z0011, International Breast Cancer Study Group (IBCSG) - 01 and After Mapping of the Axilla: Radiotherapy Or Surgery (AMAROS)) have published their long-term results, with non-inferior survival outcomes following omission of ALND in a selected group of early breast cancer (EBC) patients with metastatic SLNs [5-7].

The landmark prospective randomised controlled trial (RCT) Z0011 included EBC patients undergoing breast conservation surgery (BCS) with tumour size $<5 \mathrm{~cm}$ and up to two metastatic SLN receiving planned radiotherapy and systemic treatment. This study has randomised patients in two arms - ALND in the standard arm and no further axillary treatment in the experimental arm [8]. The authors published initial results in 2011 with 6.3 years of follow up showing equivalent survival in both arms [9]. After this publication, there was extensive debate among breast cancer experts worldwide about the applicability of the trial results in clinical practice. The study was extensively criticised, with incomplete accrual, unclear radiation fields, mainly consisting of good biology tumour and many more issues [10, 11]. Despite the criticism, the study's early results have led to significant changes in practice and guidelines, and many surgeons now omit ALND in SLN positive patients, who fulfil the trial inclusion criteria [12]. The authors published long term 10 years survival outcomes in 2018, and reported no difference between the two arms in overall survival (OS) (83.6\% versus $86.3 \%$, non-inferiority $p=0.02$ ), Disease-free survival (DFS) (78.2\% versus $80.2 \%, p=0.32)$ and locoregional relapse-free survival $(81.2 \%$ versus $83 \%, p=0.41)[6]$. The Z0011 authors reported that the ALND arm had more seroma $(p<0.001)$, paraesthesia $(p<0.001)$, wound complications $(p=0.0016)$ and lymphedema rate $(p<0.001)$ compared to the SLNB arm [13].

Several validation studies have supported the results of the Z0011 trial, mainly in Western populations with a high proportion of screendetected breast cancer [12, 14, 15]. In the United Kingdom, the ongoing RCT POSNOC (Positive Sentinel Node - Adjuvant therapy alone versus Adjuvant therapy plus Clearance or Axillary Radiotherapy) is recruiting patients undergoing either BCS or mastectomy with up to two SLN macrometastases, who are randomised to either no further axillary treatment or additional treatment (ALND or axillary radiotherapy) [16]. In Asia, data regarding the application of Z0011 results in clinical practice is minimal [17]. Apart from trial critics, oncologists in Asia are hesitant to de-escalate axillary surgery in patients with metastatic SLNs, with concerns about the comparatively advanced stage of presentation in the non-screened population [18]. In a survey of axillary management practice amongst surgeons of India, only $15.8 \%$ of the respondents have reported omission of ALND in Z0011 trial eligible patients [19]. In contrast, many oncologists from the developed countries have accepted the Z0011 results and are moving further by extrapolating Z0011 results in mastectomy patients with 1-2 metastatic SLNs [20, 21].

The objectives of the present study were to compare our population of non-screened breast cancer with the Z0011 patients in terms of demographics, histopathological characteristics and survival. In addition, we analysed the impact of additional information gained from completion ALND on decisions related to adjuvant treatment and survival.

\section{Methodology}

The retrospective study was done with approval from the Institutional Review Board (EC/WV/TMC/013/19). All clinically node-negative EBC patients who underwent SLNB from 2013 to 2017 were included. We excluded patients who had prior breast surgery, pure ductal carcinoma in situ, and SLN positive with isolated tumour cells only. Data were extracted from the prospectively maintained REDcap (Vanderbilt University, Nashville, TN) database and hospital management system. Patients follow-up data were retrieved until 30 April 2020 to identify events of interest (death and recurrence).

The SLNB was performed by using either radioisotope, methylene blue (MB) or both as per the availability of the radioisotope. Microfiltered (0.22 micron) $99 \mathrm{~m}$ Tc-S was injected at least 2 hours before surgery in the peritumoral region. $2 \mathrm{ml}$ of $1 \% \mathrm{MB}$ dye was injected in the periareolar area just before the surgery, followed by 5 minutes of whole breast massage. A Gama camera (Europrobe3, EURORAD S.A2) was used to identify the radioactive nodes. Lymph nodes with radioactive count $>10 \%$ of the tumour count were considered as hot nodes. All hot, visibly blue and clinically suspicious nodes were labelled as SLN and send for frozen section, which was reported as per AJCC and ASCO guidelines [22, 23]. All patients with positive SLNs on the frozen section or subsequent paraffin section had completion ALND. 


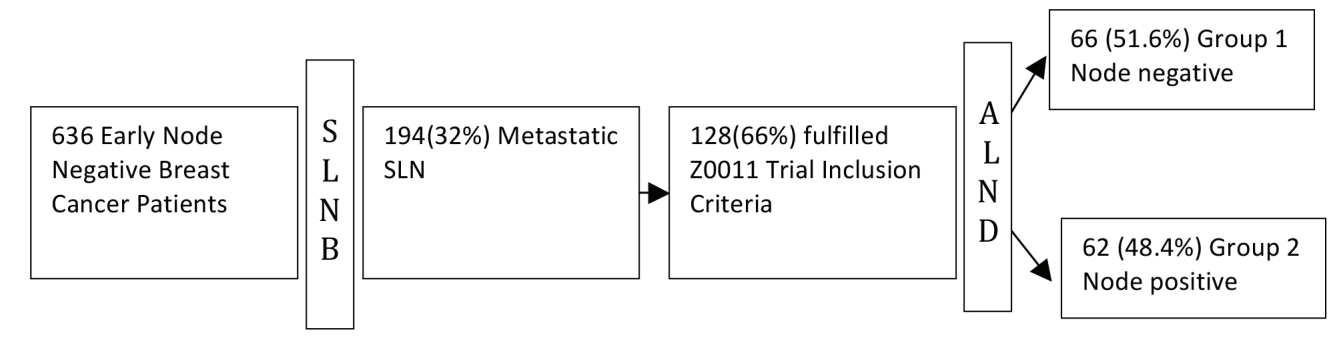

Figure 1. Patient selection (study schema).

All patients with metastatic SLNs were screened for the inclusion criteria of the Z0011 study. Patients who have fulfilled the Z0011 inclusion criteria were included in the analysis. Clinical and histopathological characters of the present cohort were compared with the Z0011 study population. All Z0011 eligible patients were divided into two groups on the basis of further metastatic nodes being identified on completion ALND - group one included patients who had no further metastatic nodes, and group two included patients with metastatic nodes (Figure 1). The possibility of extrapolation of Z0011 results to mastectomy patients was explored by including mastectomy patients with relevant tumour and SLN characteristics, with Z0011 eligible patients (Z0011 + mastectomy) as a separate analysis. The effect of further information gained by completion ALND on the stage of cancer and adjuvant treatment decisions was assessed by comparison between the two groups as defined above. The additional benefit of ALND on survival was evaluated by a comparative analysis of DFS and OS between two groups.

All patients received adjuvant treatment as per multidisciplinary tumour board recommendations. Predict NHS tool was used for prognostication, and chemotherapy was recommended to all patients with survival benefit $\geq 4 \%$ at 10 years [24]. Radiotherapy was recommended for all patients who had BCS. Post mastectomy radio therapy (PMRT) was prescribed to patients with a Cambridge index score $\geq 3$ [25]. Predict NHS tool and Cambridge index were used for decision making due to the non-availability of national cancer guidelines during time of the study. The aforementioned validated tools are easy to use in resource constraint settings. All patients were treated using linear accelerators, and treatment planning was done using CT scan based 3d-conformal techniques and details of the treatment planning procedure is available from our previous publication [26]. Tumour bed boost was given post BCS, and $2 \mathrm{~cm}$ bolus ( $1 \mathrm{~cm}$ above and below the mastectomy scar, of 5 $\mathrm{mm}$ thickness) was used in post-mastectomy patients. The axilla was not irradiated as per institutional protocol, and all patients have received breast, chest wall, and supraclavicular radiotherapy. We used a field-based technique for coverage of the chest wall and supraclavicular area. The lateral edge of the tangential field was placed such that the apex of the axilla was spared. Matched supraclavicular field radiotherapy using Case bow's technique [27] was used such that there was no overlap between supraclavicular fossa and tangential field.

Endocrine therapy was recommended to all patients with positive oestrogen receptor (Allred score $\geq 3$ ) or positive progesterone receptor (Allred score $\geq 3$ ). Trastuzumab was recommended to all HER 2 positive patients.

Summary statistics were represented by a percentage, median and interquartile range. Univariate analysis was done by Chi-square/Fisher exact test, and multivariate analysis was done with binary logistic regression. DFS was defined as the duration from the date of diagnosis to the date of recurrence (locoregional or distant), and OS as the duration from the time of diagnosis to death from any cause. Survival analysis was done by the Kaplan-Meier method, and log-rank $p$-value was used to compare the two groups. Patients who have either completed follow up without event or lost to follow up were censored. All $p$ values were two-tailed, and $p<0.05$ was considered to be statistically significant. Statistical Package for the Social Sciences version 25 (IBM Corporation, Chicago, IL) and STATA version 15 (College Station, TX: Stata Corp LLC) were used for analysis.

\section{Results}

636 patients had SLNB during the study period, and SLNs were identified in 606 patients, with an identification rate of $95.2 \%$. 194/606 (32\%) patients had metastatic SLN. 128/194 (66\%) patients fulfilled the inclusion criteria of the Z0011 study (Figure 1). The patients were divided into two groups as per metastatic lymph nodes in completion ALND - 66 (51.6\%) had no further positive nodes (Group 1), and 62 (48.4\%) had additional metastatic nodes (Group 2). 
The median age was 51 years (IQR 44, 59), and the median tumour size was $2.5 \mathrm{~cm}$ (IQR 2.2, 3.2). Table 1 compares the demographic, clinical and histopathological characteristics of our Z0011 eligible cohort with participants from the original study. We found significant differences, with our group being younger and with more aggressive disease (higher proportions of invasive ductal cancer, T2, grade 3 and progesterone receptor negativity). The SLNs had macro metastasis in $82.8 \%$ of patients versus $58.8 \%$ in the Z0011 study. Following completion of ALND, further metastatic nodes were identified in 48\%, compared to $27 \%$ in the ALND arm of Z0011. In Table 2, we have summarised factors associated with further nodal metastasis in the completion ALND. The tumour stage (T2 > T1) and the SLN macro metastasis were significantly associated with further nodal metastases.

Table 1. Clinicopathological characteristics of study versus Z0011 population.

\begin{tabular}{|c|c|c|c|c|}
\hline Parameters & $\begin{array}{l}\text { Z0011 study } \\
\text { intention to treat } \\
(n=856)\end{array}$ & $\begin{array}{l}\text { Present study } \\
\quad(n=128)\end{array}$ & $X^{2}$ & $p$ \\
\hline \multicolumn{5}{|l|}{ Age, years } \\
\hline$\leq 50$, no. (\%) & $295(35.2)$ & $62(48.4)$ & 7.84 & 0.005 \\
\hline$>50$, no. (\%) & $544(64.8)$ & $66(51.6)$ & & \\
\hline \multicolumn{5}{|l|}{ Clinical T stage, no. (\%) } \\
\hline T1 & 587 (69.3) & $22(17.2)$ & 126.8 & $<0.001$ \\
\hline $\mathrm{T} 2$ & $260(30.7)$ & $106(82.8)$ & & \\
\hline \multicolumn{5}{|c|}{ Estrogen receptor, no. (\%) } \\
\hline $\mathrm{ER}+$ & 659 (83) & 108 (84.4) & 0.14 & $0.69, n s$ \\
\hline ER- & 135 (17) & $20(15.6)$ & & \\
\hline \multicolumn{5}{|c|}{ Progesterone receptor, no. (\%) } \\
\hline $\mathrm{PR}+$ & $534(68.8)$ & $105(82)$ & 9.2 & 0.003 \\
\hline PR- & $242(31.2)$ & $23(18)$ & & \\
\hline \multicolumn{5}{|c|}{$\begin{array}{l}\text { Modified Bloom-Richardson } \\
\text { score, no (\%) }\end{array}$} \\
\hline Grade 1 & $152(23.8)$ & $9(7)$ & 31.7 & $<0.001$ \\
\hline Grade 2 & 306 (47.9) & $54(42.2)$ & & \\
\hline Grade 3 & $181(28.3)$ & $65(50.8)$ & & \\
\hline \multicolumn{5}{|l|}{ Tumor type, no. (\%) } \\
\hline Infiltrating ductal & 700 (80.3) & $120(93.8)$ & 9.7 & $<0.001$ \\
\hline Infiltrating lobular & $63(7.5)$ & $5(3.9)$ & & \\
\hline Others & $77(9.2)$ & $3(2.3)$ & & \\
\hline \multicolumn{5}{|c|}{ Size of SLN mets, no. (\%) } \\
\hline Micrometastasis & $301(41.2)$ & $22(17.2)$ & 25.7 & $<0.001$ \\
\hline Macrometastasis & $430(58.8)$ & $106(82.8)$ & & \\
\hline Parameters & $\begin{array}{c}\text { Z0011 ALND } \\
\text { arm, intention to } \\
\text { treat }(n=420)\end{array}$ & $\begin{array}{l}\text { Present study } \\
\quad(n=128)\end{array}$ & $X^{2}$ & $p$ \\
\hline \multicolumn{5}{|c|}{ Completion ALND, no. (\%) } \\
\hline Non SLN positive & $97(27)$ & $62(48.4)$ & 18.04 & $<0.001$ \\
\hline Non SLN negative & $258(73)$ & $66(51.6)$ & & \\
\hline
\end{tabular}

$\mathrm{ER}=$ Estrogen receptor, $\mathrm{PR}=$ Progesterone receptor, $\mathrm{ALND}=$ Axillary lymph node dissection, $\mathrm{SLN}=$ Sentinel lymph node 
Table 2. Clinicopathological characteristics of Group 1 (node negative ALND) versus Group 2 (node positive ALND).

\begin{tabular}{|c|c|c|c|c|c|}
\hline Parameters & $\begin{array}{l}\text { Group } 1 \text { (node } \\
\text { negative ALND) } \\
\quad(n=66)\end{array}$ & $\begin{array}{c}\text { Group } 2 \text { (node } \\
\text { positive ALND) } \\
(n=62)\end{array}$ & $\mathrm{X}^{2}$ & $p$ (univariate) & $p$ (multivariate) \\
\hline \multicolumn{6}{|l|}{ Age, years } \\
\hline$\leq 50$, no. (\%) & $32(48.5)$ & $30(48.4)$ & 0.00 & $1.00, n s$ & $0.77, n s$ \\
\hline$>50$, no. (\%) & $34(51.5)$ & $32(51.6)$ & & & \\
\hline \multicolumn{6}{|l|}{ Clinical T stage,no. (\%) } \\
\hline $\mathrm{T} 1$ & $17(25.8)$ & $5(8.1)$ & 7.03 & 0.01 & 0.02 \\
\hline $\mathrm{T} 2$ & $49(74.2)$ & $57(91.9)$ & & & \\
\hline \multicolumn{6}{|l|}{ Estrogen receptor, no. (\%) } \\
\hline ER+ & $54(81.8)$ & $54(87.1)$ & 0.67 & $0.47, \mathrm{~ns}$ & $0.16, n s$ \\
\hline ER- & $12(18.2)$ & $8(12.9)$ & & & \\
\hline \multicolumn{6}{|l|}{ Progesterone receptor, no. (\%) } \\
\hline $\mathrm{PR}+$ & $51(77.3)$ & $54(87.1)$ & 2.09 & $0.17, n s$ & $0.17, n s$ \\
\hline PR- & $15(22.7)$ & $8(12.9)$ & & & \\
\hline \multicolumn{6}{|c|}{ Modified Bloom-Richardson score, no (\%) } \\
\hline Grade 1 & $2(3)$ & $7(11.3)$ & & $\begin{array}{c}0.18 \\
\text { (Fisher exact test), ns }\end{array}$ & $0.15, n s$ \\
\hline Grade 2 & $30(45.5)$ & $24(38.7)$ & & & \\
\hline Grade 3 & $34(51.5)$ & $31(50)$ & & & \\
\hline \multicolumn{6}{|l|}{ Tumor type, no. (\%) } \\
\hline Infiltrating ductal & $63(95.5)$ & $57(91.9)$ & & $\begin{array}{c}0.39 \\
\text { (Fisher exact test), ns }\end{array}$ & $0.26, n s$ \\
\hline Infiltrating lobular & $1(1.5)$ & $4(6.5)$ & & & \\
\hline Others & $2(3)$ & $1(1.6)$ & & & \\
\hline \multicolumn{6}{|l|}{ Size of SLN mets, no. (\%) } \\
\hline Micrometastasis & $20(30.3)$ & $2(3.2)$ & 16.46 & $<0.001$ & 0.001 \\
\hline Macrometastasis & $46(69.7)$ & $60(96.8)$ & & & \\
\hline
\end{tabular}

$E R=$ Estrogen receptor, $\mathrm{PR}=$ Progesterone receptor, $\mathrm{ALND}=$ Axillary lymph node dissection, $\mathrm{SLN}=$ Sentinel lymph node

Table 3 summarises the impact of further nodal positivity by completion ALND on tumour stage and adjuvant treatment decision compared to SLNB only. Although the tumour and nodal stage were upgraded in 19.5\% of our Z0011 eligible cohort, multidisciplinary team (MDT) recommendations for chemotherapy were changed in only $3 \%$ of patients. In the Z0011 + mastectomy cohort, the tumour and nodal stage were upgraded in $18.7 \%$, with chemotherapy recommendations being changed in $2.2 \%$. PMRT decisions did not change for any patients. Only $5 / 48$ (10\%) of mastectomy patients with low-risk features (T1N1, G1-2) were recommended no radiotherapy as per the Cambridge Index score.

As per our records, in Z0011 eligible patients, $96 \%$ and $94 \%$ of patients have received recommended chemotherapy and radiotherapy, respectively. The HER 2 neu receptor status was known for 124/128 Z0011 eligible patients. 22/124 (17.7\%) were HER 2 receptor-positive, and only $8 / 22$ (36\%) have received trastuzumab due to financial constraints. The compliance to endocrine therapy was good in our cohort, and $>95 \%$ of the patients have followed prescribed hormonal treatment at the time of data collection.

The median follows up was 40 (IQR 29, 54) months. In the Z0011 eligible cohort ( $n=128)$, there were eight recurrences (six distant, two locoregional) and eight deaths (five disease progression, one accidental, one leukaemia and one myocardial infarction). The 5 years estimated OS was $92.8 \%$, and DFS was $92.6 \%$. Further nodal positivity in completion ALND was not significantly associated with OS ( $p=0.86$, Figure $2 \mathrm{a}$ ) and DFS ( $p=0.5$, Figure $2 \mathrm{~b}$ ). 
Table 3. Stage and adjuvant treatment decision between SLNB (only) versus SLNB + ALND.

\begin{tabular}{|c|c|c|c|}
\hline \multicolumn{4}{|c|}{ Z0011 eligible patients, $n=128$} \\
\hline Parameters & SLNB only & SLNB + ALND & Remarks \\
\hline \multicolumn{4}{|l|}{ Nodal stage, $n(\%)$} \\
\hline N1 & $128(100)$ & $103(80.5)$ & Nodal stage changed in $19.5 \%$. \\
\hline N2 & 0 & $24(18.8)$ & \\
\hline N3 & 0 & $1(0.8)$ & \\
\hline \multicolumn{4}{|l|}{ TNM stage (AJCC 7th), $n$ (\%) } \\
\hline Stage 2 & $128(100)$ & $103(80.5)$ & TNM stage changed in $19.5 \%$. \\
\hline Stage 3 & 0 & $25(19.5)$ & \\
\hline \multicolumn{4}{|c|}{ MDT decision (chemotherapy), $n$ (\%) } \\
\hline Yes & $115(89.8)$ & $119(93)$ & $\begin{array}{l}\text { In } 4 / 128 \text { (3\%), ALND changed the } \\
\text { decision of chemotherapy. }\end{array}$ \\
\hline No & $13(10.2)$ & $9(7)$ & \\
\hline \multicolumn{4}{|c|}{ Z0011 eligible + mastectomy, $n=176$} \\
\hline \multicolumn{4}{|l|}{ Nodal stage, $n(\%)$} \\
\hline N1 & $176(100)$ & $143(81.3)$ & Nodal stage changed in $18.7 \%$. \\
\hline N2 & 0 & $28(15.9)$ & \\
\hline N3 & 0 & $5(2.8)$ & \\
\hline \multicolumn{4}{|l|}{ TNM stage (AJCC 7th), n (\%) } \\
\hline Stage 2 & $176(100)$ & $143(81.3)$ & TNM stage changed in $18.7 \%$. \\
\hline Stage 3 & 0 & $33(18.7)$ & \\
\hline \multicolumn{4}{|c|}{ MDT decision (chemotherapy), $n$ (\%) } \\
\hline Yes & $157(89.8)$ & $161(91.5)$ & $\begin{array}{l}\text { In } 4 / 176 \text { ( } 2.2 \%) \text {, ALND changed the } \\
\text { decision of chemotherapy. }\end{array}$ \\
\hline No & $19(10.2)$ & $15(8.5)$ & \\
\hline \multicolumn{4}{|c|}{ MDT decision (Radiotherapy), $n=48, n$ (\%) } \\
\hline Yes & $43(89.6)$ & $43(89.6)$ & No change \\
\hline No & $5(10.4)$ & $5(10.4)$ & \\
\hline
\end{tabular}

SLNB = Sentinel lymph node biopsy, ALND = Axillary lymph node biopsy, MDT = Multi-disciplinary team

In Z0011 eligible + mastectomy cohort $(n=176)$, there were 12 recurrences (10 distant, 2 locoregional) and 12 deaths (9 disease progression, 1 accidental, 1 leukaemia and 1 myocardial infarction). The 5 years estimated OS and DFS were 93.1\%. Further nodal positivity in completion ALND was not significantly associated with OS ( $p=0.63$, Figure $3 a$ ) and DFS ( $p=0.87$, Figure $3 b)$.

\section{Discussion}

In India at present, the omission of ALND in Z0011 eligible patients, in general, is not a routine practice [18], so it is difficult to make a direct assessment of the impact of ALND following positive SLNB. To compare the outcome of these two approaches, we chose a group of patients who would fit the Z0011 study inclusion criteria, but all of whom had ALND. We then divided the study cohort into two groups depending upon further nodal positivity in the histopathology report of ALND (Figure 1). The comparative analysis between two groups (node-negative versus node-positive in completion ALND) enabled us to estimate the impact of non-sentinel nodal metastasis on adjuvant treatment and survival. 


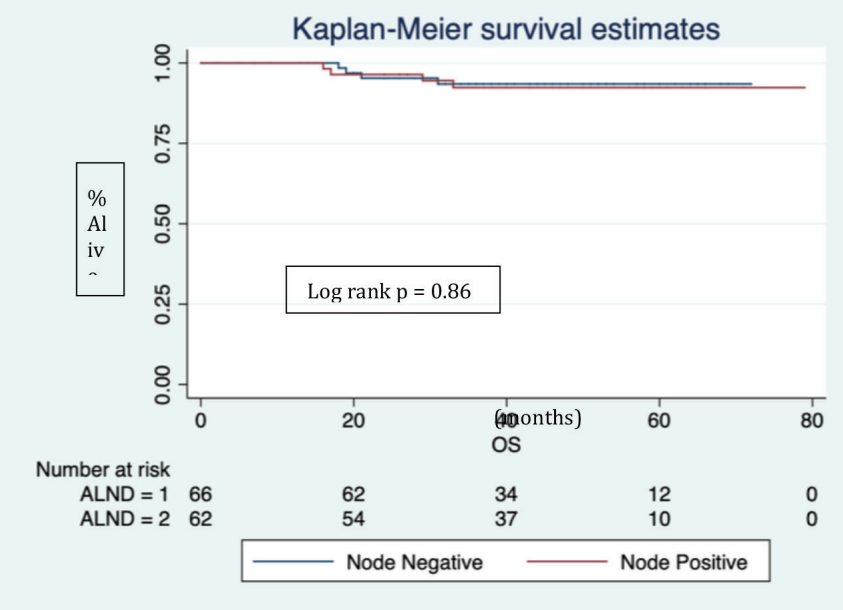

(a)

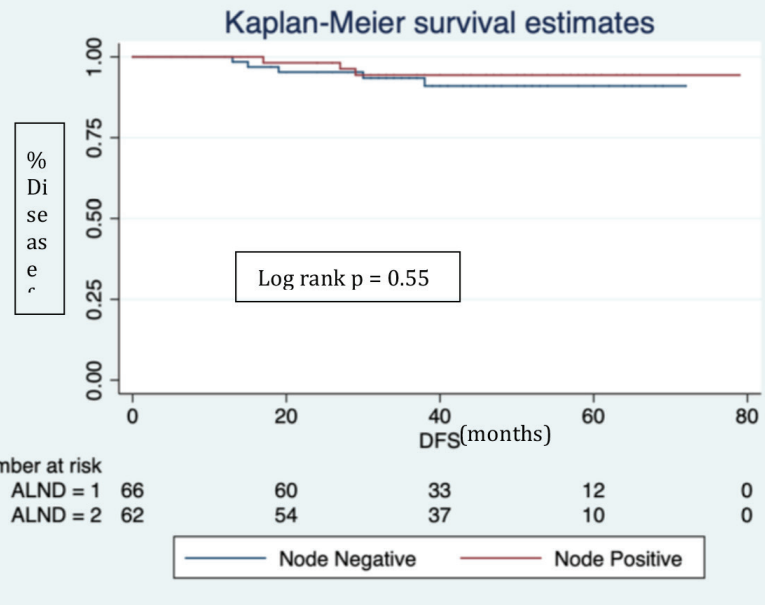

(b)

Figure 2. Overall and disease-free survival in Z0011 eligible patients.

$(A L N D=1)$ represents node negative and $(A L N D=2)$ node positive in completion ALND.

In a median follow up of 40 (IQR 29, 54) months.

(a): Four deaths in each ALND group (1\&2).

(b): Five recurrences in ALND (node negative) and three in ALND (node positive).

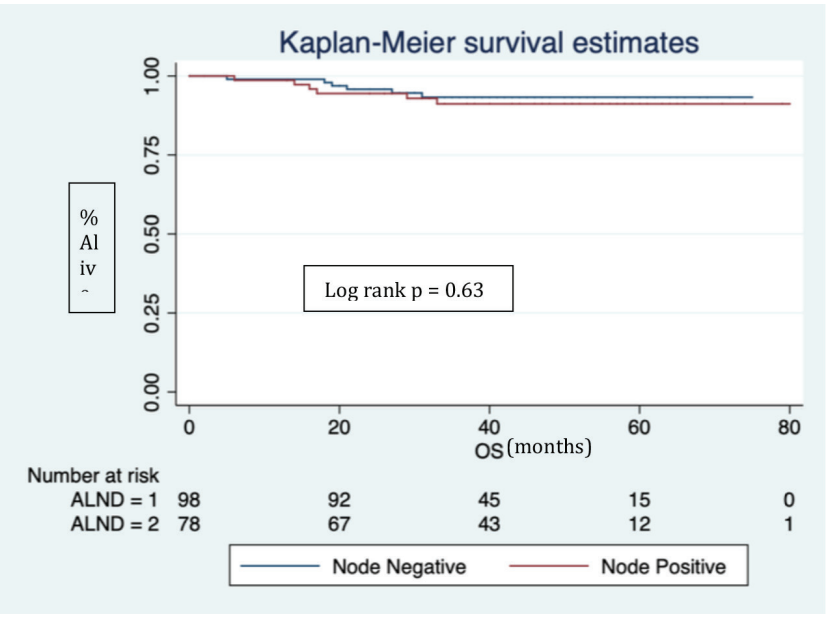

(a)

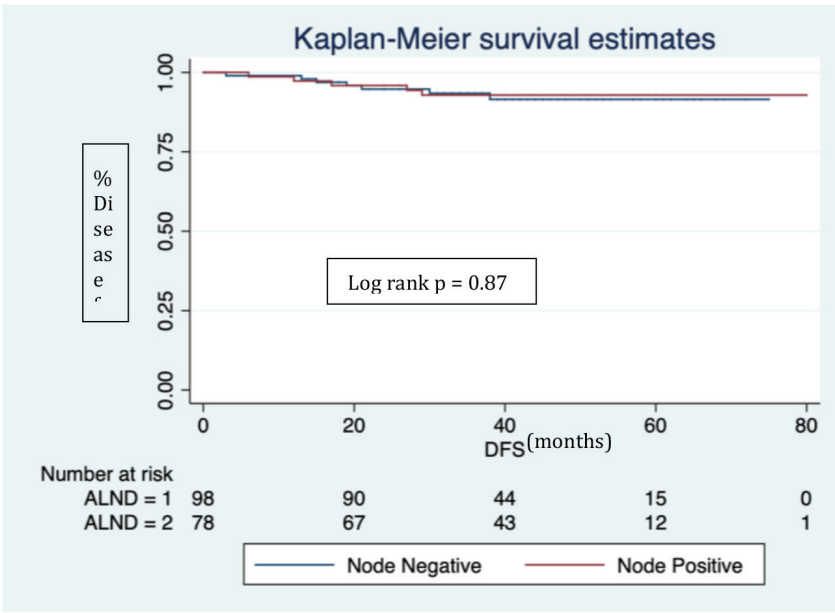

(b)

Figure 3. Overall and disease-free survival in Z0011 eligible patients + mastectomy.

$(A L N D=1)$ represents node negative and $(A L N D=2)$ node positive in completion ALND.

In a median follow up of $40($ IQR 29,54$)$ months:

(a): Six deaths in each ALND group (1\&2).

(b): Seven recurrence in ALND (node negative) and five in ALND (node positive).

Compared to Z0011 study participants, EBC patients in our cohort had significantly larger tumours, more aggressive biology, higher SLN macrometastasis rate and more nodal metastasis in completion ALND. The difference in tumour characteristics may be due to the lack of a breast cancer screening program in India, where patients generally seek physician's advice for breast cancer symptoms like lump or nipple 
discharge [28]. Additionally, breast cancer in India presents more commonly in young women with aggressive biology such as G3 and triple negative [29]. The higher stage of presentation and aggressive biology are the primary concerns of the oncologist in India for omitting ALND in Z0011 eligible patients. Besides, there is a fear that omission of ALND may leave residual pathological nodes, which in turn may lead to poorer survival [18]. A National Cancer Data Base study published similar concerns in experts from the western region and have concluded that younger patients and those with HER2 positive and triple-negative subtype were more likely to undergo ALND even though fulfilling eligibility criteria's of the Z0011 study [30]. However, another recently published study has highlighted overtreatment for EBC patients in India, resulting in patients facing unnecessary financial burdens, treatment-related complications and reduced quality of life [31].

In our study, SLNs were the only metastatic node in $51.6 \%$ of patients. T2 stage and macro metastasis in SLN were factors significantly associated with the finding of further metastatic nodes in completion ALND. The aggressive tumour characters like high grade, ER negativity and younger age were not significantly associated with the residual axillary burden. Similar results of lack of association of aggressive tumour biology and non-SLN positivity in Z0011 eligible patients was reported in multiple studies [32, 33]. The non-association of aggressive tumour characteristics with residual axillary burden supports omission of completion ALND in patients fulfilling Z0011 eligibility criteria.

Systemic therapy in EBC patients is now, in general, decided by tumour biology and genomic predictors rather than regional nodal status $[34,35]$. In combination with clinical and histopathological characteristics by using predict NHS tool [24], 89\% of our patients were advised adjuvant chemotherapy based on information derived from the SLNB result alone. With additional information available from ALND, a further four patients (3\% overall) were also recommended chemotherapy. Thus, after the MDT recommendation, $86 \%$ of our patients had chemotherapy, which is substantially higher than the Z0011 study population, in which 58\% had received chemotherapy in SLNB and $57.9 \%$ in ALND arm [8].

Several studies have examined whether it is possible to extrapolate the results of Z0011 to mastectomy patients. However, some authors have raised concerns that information of additional positive nodes in ALND may play a crucial role in decision making for radiotherapy [36]. In our Z0011 + mastectomy cohort, we found that the additional information from completion ALND did not change the radiotherapy recommendation for any patient. All Z0011 + mastectomy patients in our cohort were mainly T1-2, N1 breast cancer patients and the role of PMRT improving survival in this group is supported by level one evidence like EBCTCG metanalysis [37].

The 5-year estimated OS in the present cohort was $92.6 \%$, which is similar to the Z0011 study, which reported a 5-year OS rate of $92.5 \%$ for SLNB group and $91.8 \%$ in ALND group [8]. In spite of the relatively advanced stage of presentation, in our cohort, the 5-year estimated DFS was $92.6 \%$ which is better than the Z0011 study, which reported 5 -year DFS of $83.9 \%$ in the SLNB group and $82.2 \%$ in the ALND group. The lower rate of distant recurrences may be attributed to the higher proportions of patients receiving chemotherapy in our cohort (86\% versus 58\%) [8]. Additionally, in our cohort, 83\% of patients received endocrine therapy compared to $46 \%$ in the Z0011 study population [8]. When we compared survival between additional node-negative and node-positive patients following completion ALND, there was no significant difference in OS and DFS. The absence of survival differences between the two groups may indicate that it may be appropriate to avoid ALND in these patients, and systemic therapies may be adequate to take care of additional nodal metastases.

The approach to patients with positive SLNs is gradually changing. The St. Gallen consensus (2019) panel has recommended avoiding ALND if regional nodal irradiation is planned for post-mastectomy patients who otherwise fulfil Z0011 eligibility criteria [34]. Another comparative analysis of mastectomy and BCS patients who had metastatic SLNs without completion ALND showed equivalent survival results, irrespective of the type of surgery, with 4-year DFS and OS of $94.8 \%$ and $97.8 \%$ in the mastectomy group versus $90.1 \%$ and $92.6 \%$ in the BCS group [38]. In our study too, the 5-year estimated OS and DFS were almost the same in Z0011 + mastectomy patients compared to Z0011 eligible BCS patients. These results suggest that it may be possible to extrapolate Z0011 results to mastectomy patients with tumours $<5 \mathrm{~cm}$ and 1-2 metastatic SLNs, to avoid the morbidity associated with ALND.

Our study has some potential limitations. It is a retrospective data analysis, which may have some selection biases. At the time of analysis, the follow-up time was $40($ IQR 29,54$)$ months with a low event rate, which may contribute to no significant difference in survival between the two groups. Longer-term follows up with more events may adequately power the study to see any difference in survival between two groups. The majority of HER 2 receptor-positive patients in our cohort have not received trastuzumab, which may affect the survival analysis. The chemotherapy decisions were based on clinical risk predictors based on NHS predict score, and it may change by using genomic scores in the current practice. 


\section{Conclusion}

In conclusion, Z0011 eligible Indian EBC patients are significantly different compared to the Z0011 study population, with younger age of presentation, higher grade, a higher rate of both SLN macro metastasis and non-SLN positivity. Despite these aggressive characters, the incremental impact of completion ALND on adjuvant chemotherapy and radiotherapy decisions change was minimal. The non-sentinel metastasis was not associated with poor survival in present cohort. These results may give food for thought to LMIC oncologists to avoid completion ALND in EBC patients (BCS with $T<5 \mathrm{~cm}, 1-2$ positive SLN).

\section{Conflicts of interest}

The authors declare no conflicts of interest.

\section{Funding}

There was no funding received for this study.

\section{List of abbreviations}

ALND, Axillary lymph node dissection; DFS, Disease-free survival; EBC, Early breast cancer; NCDB, National Cancer Data Base; OS, Overall survival; PMRT, Post mastectomy radio therapy; RCT, Randomised controlled trial; SLN, Sentinel lymph node; SLNB, Sentinel lymph node biopsy.

\section{Authors' contributions}

Conceptualisation: Sanjit Kumar Agrawal; methodology: Sanjit Kumar Agrawal; data collection: Vishal Kewlani, Noopur Priya, Sanjit Kumar Agrawal, Rosina Ahmed; data analysis: Vishal Kewlani, Sanjit Kumar Agrawal; writing - original draft preparation: Sanjit Kumar Agrawal; writing - review and editing: all authors; final approval of manuscript: all authors.

\section{References}

1. Mansel RE, Fallowfield L, and Kissin M, et al (2006) Randomized multicenter trial of sentinel node biopsy versus standard axillary treatment in operable breast cancer: the ALMANAC trial JNCI J Natl Cancer Inst 98(9) 599-609 https://doi.org/10.1093/jnci/djj158 PMID: 16670385

2. Krag D (2007) National surgical adjuvant breast and bowel project, technical outcomes of sentinel-lymph-node resection and conventional axillary-lymph-node dissection in patients with clinically node-negative breast cancer : results from the NSABP B-32 randomised phase III trial Lancet Oncol 8 881-888 https://doi.org/10.1016/S1470-2045(07)70278-4

3. Boughey JC, Suman VJ, and Mittendorf EA, et al (2013) Sentinel lymph node surgery after neoadjuvant chemotherapy in patients with node-positive breast cancer: The American College of Surgeons Oncology Group (ACOSOG) Z1071 clinical trial JAMA J Am Med Assoc 310(14) 1455-1461 https://doi.org/10.1001/jama.2013.278932 
4. Kuehn T, Bauerfeind I, and Fehm T, et al (2013) Sentinel-lymph-node biopsy in patients with breast cancer before and after neoadjuvant chemotherapy (SENTINA): a prospective, multicentre cohort study Lancet Oncol 14(7) 609-618 https://doi.org/10.1016/S14702045(13)70166-9 PMID: 23683750

5. Donker M, van Tienhoven G, and Straver ME, et al (2014) Radiotherapy or surgery of the axilla after a positive sentinel node in breast cancer (EORTC 10981-22023 AMAROS): a randomised, multicentre, open-label, phase 3 non-inferiority trial Lancet Oncol 15(12) 1303-1310 https://doi.org/10.1016/S1470-2045(14)70460-7

6. Giuliano AE, Ballman KV, and McCall L, et al (2017) Effect of axillary sissection vs no axillary dissection on 10-year overall survival among women with invasive breast cancer and sentinel node metastasis: the ACOSOG Z0011 (alliance) randomized clinical trial JAMA 318(10) 918-926 https://doi.org/10.1001/jama.2017.11470 PMID: 28898379 PMCID: 5672806

7. Galimberti V, Cole BF, and Viale G, et al (2018) Axillary dissection versus no axillary dissection in patients with breast cancer and sentinel-node micrometastases (IBCSG 23-01): 10-year follow-up of a randomised, controlled phase 3 trial Lancet Oncol 19(10) 1385-1393 https://doi.org/10.1016/S1470-2045(18)30380-2 PMID: 30196031

8. Giuliano AE, McCall L, and Beitsch P, et al (2010) Locoregional recurrence after sentinel lymph node dissection with or without axillary dissection in patients with sentinel lymph node metastases: the American College of Surgeons Oncology Group Z0011 randomized trial Ann Surg 252(3) 426-432 https://doi.org/10.1097/SLA.0b013e3181f08f32

9. Giuliano $\mathrm{AE}$, Hunt $\mathrm{KK}$, and Ballman $\mathrm{KV}$, et al (2011) Axillary dissection vs no axillary dissection in women with invasive breast cancer and sentinel node metastasis: a randomized clinical trial JAMA 305(6) 569-575 https://doi.org/10.1001/jama.2011.90 PMID: 21304082 PMCID: 5389857

10. Zellars RC (2014) New information prompts old question: is sentinel lymph node sampling equivalent to axillary lymph node dissection? J Clin Oncol 32(32) 3583-3585 https://doi.org/10.1200/JCO.2014.57.9946 PMID: 25267737

11. Jagsi R, Chadha M, and Moni J, et al (2014) Radiation field design in the ACOSOG Z0011 (alliance) trial J Clin Oncol Off J Am Soc Clin Oncol 32(32) 3600-3606 https://doi.org/10.1200/JCO.2014.56.5838

12. Gainer SM, Hunt KK, and Beitsch P, et al (2012) Changing behavior in clinical practice in response to the ACOSOG Z0011 trial: a survey of the American Society of Breast Surgeons Ann Surg Oncol 19(10) 3152-3158 https://doi.org/10.1245/s10434-012-2523-z

13. Lucci A, McCall LM, and Beitsch PD, et al (2007) Surgical complications associated with sentinel lymph node dissection (SLND) plus axillary lymph node dissection compared with SLND alone in the American College of Surgeons Oncology Group Trial Z0011 J Clin Oncol Off J Am Soc Clin Oncol 25(24) 3657-3663 https://doi.org/10.1200/JCO.2006.07.4062

14. Morrow M, Van Zee KJ, and Patil S, et al (2017) Axillary dissection and nodal irradiation can be avoided for most node-positive Z0011-eligible breast cancers: a prospective validation study of 793 patients Ann Surg 266(3) 457-462 https://doi.org/10.1097/ SLA.0000000000002354 PMID: 28650355 PMCID: 5649371

15. Ngui NK, Hitos K, and Hughes TMD (2019) Effect of the American College of Surgeons Oncology Group Z0011 trial on axillary management in breast cancer patients in the Australian setting Breast J 25(5) 853-858 https://doi.org/10.1111/tbj.13343

16. Goyal A, Mann B, and Thompson AM (2018) POSNOC: positive sentinel node-adjuvant therapy alone versus adjuvant therapy plus clearance or axillary radiotherapy J Clin Oncol 36(15_suppl) TPS600-TPS600 https://doi.org/10.1200/JCO.2018.36.15_suppl.TPS600

17. Jung J, Kim BH, and Kim J, et al (2020) Validating the ACOSOG Z0011 trial result: a population-based study using the SEER database Cancers 12(4) 950 https://doi.org/10.3390/cancers12040950 PMCID: 7226449

18. Chatterjee S, Agrawal S, and Nallathambi C, et al (2017) Is the current American Society of Clinical Oncology Guidance on axillary management generalisable for symptomatic or unscreened breast cancers? Clin Oncol R Coll Radiol G B 29(10) e172-e173 https://doi. org/10.1016/j.clon.2017.06.004 
19. Agrawal SK, Priya N, and Agarwal P, et al (2021) Trends in axillary management of early breast cancer: a questionnaire-based pattern of practice survey for India Indian J Surg Oncol 12(2) 401-407 https://doi.org/10.1007/s13193-021-01334-4 PMID: 34295086 PMCID: 8272771

20. Tc K, J D, and M S, et al (2016) Widespread implications of ACOSOG Z0011: effect on total mastectomy patients Am Surg 82(1) 53-58 https://doi.org/10.1177/000313481608200124

21. Arisio R, Borella F, and Porpiglia M, et al (2019) Axillary dissection vs. no axillary dissection in breast cancer patients with positive sentinel lymph node: a single institution experience In Vivo 33(6) 1941-1947 https://doi.org/10.21873/invivo.11689 PMID: 31662523 PMCID: 6899081

22. Giuliano AE, Connolly JL, and Edge SB, et al (2017) Breast cancer-major changes in the American Joint Committee on cancer eighth edition cancer staging manual CA Cancer J Clin 67(4) 290-303 https://doi.org/10.3322/caac.21393

23. Lyman GH, Somerfield MR, and Bosserman LD, et al (2017) Sentinel lymph node biopsy for patients with early-stage breast cancer: American Society of Clinical Oncology clinical practice guideline update J Clin Oncol Off J Am Soc Clin Oncol 35(5) 561-564 https://doi. org/10.1200/JCO.2016.71.0947

24. Gray E, Marti J, and Brewster DH, et al (2018) Independent validation of the PREDICT breast cancer prognosis prediction tool in 45,789 patients using Scottish Cancer Registry data Br J Cancer 119(7) 808-814 https://doi.org/10.1038/s41416-018-0256-x PMID: 30220705 PMCID: 6189179

25. Mukesh MB, Duke S, and Parashar D, et al (2014) The Cambridge post-mastectomy radiotherapy (C-PMRT) index: a practical tool for patient selection Radiother Oncol 110(3) 461-466 https://doi.org/10.1016/j.radonc.2013.09.024 PMID: 24424386

26. Chatterjee S, Arunsingh M, and Agrawal S, et al (2016) Outcomes following a moderately hypofractionated adjuvant radiation (START B Type) schedule for breast cancer in an unscreened non-caucasian population Clin Oncol 28(10) e165-e172 https://doi.org/10.1016/j. clon.2016.05.008

27. Chatterjee S, Chakraborty S, and HYPORT Adjuvant Author Group (2020) Hypofractionated radiation therapy comparing a standard radiotherapy schedule (over 3 weeks) with a novel 1-week schedule in adjuvant breast cancer: an open-label randomized controlled study (HYPORT-Adjuvant)-study protocol for a multicentre, randomized phase III trial Trials 21(1) 819 https://doi.org/10.1186/s13063020-04751-y PMID: 32998747 PMCID: 7526182

28. Singh S, Shrivastava JP, and Dwivedi A (2015) Breast cancer screening existence in India: a nonexisting reality Indian J Med Paediatr Oncol Off J Indian Soc Med Paediatr Oncol 36(4) 207-209 https://doi.org/10.4103/0971-5851.171539

29. Sathwara JA, Balasubramaniam G, and Bobdey SC, et al (2017) Sociodemographic factors and late-stage diagnosis of breast cancer in India: a hospital-based study Indian J Med Paediatr Oncol 38(3) 277 https://doi.org/10.4103/ijmpo.ijmpo_15_16 PMID: 29200673 PMCID: 5686966

30. Ong CT, Thomas SM, and Blitzblau RC, et al (2017) Patient age and tumor subtype predict the extent of axillary surgery among breast cancer patients eligible for the American College of Surgeons Oncology Group Trial Z0011 Ann Surg Oncol 24(12) 3559-3566 https:// doi.org/10.1245/s10434-017-6075-0 PMID: 28879416 PMCID: 5994237

31. Bhattacharyya GS, Doval DC, and Desai CJ, et al (2020) Overview of breast cancer and implications of overtreatment of early-stage breast cancer: an Indian perspective JCO Glob Oncol 6(6) 789-798 https://doi.org/10.1200/G0.20.00033 PMID: 32511068 PMCID: 7328098

32. Loza CM, Mandó P, and Ponce C, et al (2018) Predictive factors for non-sentinel lymph node metastasis in patients with ACOSOG Z0011 criteria Breast Care 13(6) 434-438 https://doi.org/10.1159/000488277

33. Eldweny H, Alkhaldy K, and Alsaleh N, et al (2012) Predictors of non-sentinel lymph node metastasis in breast cancer patients with positive sentinel lymph node (pilot study) J Egypt Natl Cancer Inst 24(1) 23-30 https://doi.org/10.1016/j.jnci.2011.12.004 
34. Balic M, Thomssen C, and Würstlein R, et al (2019) St. Gallen/Vienna 2019: a brief summary of the consensus discussion on the optimal primary breast cancer treatment Breast Care 14(2) 103-110 https://doi.org/10.1159/000499931 PMID: 31798382 PMCID: 6886108

35. Vieira AF, and Schmitt F (2018) An update on breast cancer multigene prognostic tests-emergent clinical biomarkers Front Med 5248 https://doi.org/10.3389/fmed.2018.00248

36. Yi M, Kuerer HM, and Mittendorf EA, et al (2013) Impact of the ACOSOG Z0011 criteria applied to a contemporary patient population J Am Coll Surg 216(1) 105-113 https://doi.org/10.1016/j.jamcollsurg.2012.09.005

37. EBCTCG (Early Breast Cancer Trialists' Collaborative Group), McGale P, and Taylor C et al (2014) Effect of radiotherapy after mastectomy and axillary surgery on 10-year recurrence and 20-year breast cancer mortality: meta-analysis of individual patient data for 8135 women in 22 randomised trials The Lancet 383(9935) 2127-2135 https://doi.org/10.1016/S0140-6736(14)60488-8

38. Milgrom S, Cody H, and Tan L, et al (2012) Characteristics and outcomes of sentinel node-positive breast cancer patients after total mastectomy without axillary-specific treatment Ann Surg Oncol 19(12) 3762-3770 https://doi.org/10.1245/s10434-012-2386-3 PMID: 22576064 